\title{
A high-power lithium niobate ultrasonic transducer design for ultrasonic oil production
}

\author{
Kairan Zhang ${ }^{1, \text { a }}$ \\ ${ }^{1}$ Zhejiang University, Hangzhou 300037, China \\ akairan0512@icloud.com
}

Keywords: finite element; lithium niobate crystal; high-power ultrasonic oil production technique.

\begin{abstract}
Currently, most countries and regions often use chemical method to increase oil production of oil well in the middle and later stages. Although some results have been obtained in field application, the disadvantages of this method are obvious-the long-term use of polymer chemicals, which not only directly leads to oil reservoir pollution and desertification of land, but also reduces oil production. Due to the strong piezoelectric effect possessed by lithium niobate crystal, a new idea that uses lithium niobate to design high-power ultrasonic transducer for ultrasonic oil production technology is proposed in this paper. Firstly, the structure of this highpower is introduced. Harmonic analysis of finite element proves that such designed structure is reasonable, furthermore, two main two important parameters of ultrasonic transducers-Mechanical quality factor and Effective electromechanical coupling factor are given. The purpose of this paper is to lay the foundation for the further research and development of high-power ultrasonic oil production technique.
\end{abstract}

\section{Introduction}

According to reference [1], ultrasonic technique is one of the most promising methods for enhanced oil recovery. The effect of ultrasound on the well and the reservoir is based on two aspects of sonication that are relevant (1) reduction of the viscosity of the oil that would make it easier to pump and (2) enhancement of the flow of oil through the rocks into the pumping pool and. As one of method of physical EOR, ultrasonic oil production technique has many advantages, such as, being simple to operate, strong adaptability, low cost and no pollution to oil reservoir [2].

Thermal-based EOR techniques include steam flooding, cyclic steam injection etc. Even though these methods having direct impact on the viscosity of crude oil, the use of them for long term production of heavy oil, usually, damages the formation mainly due to deposition of paraffin near the well bore and incursion of outside liquids and solids for various types of production operations[3].

Ultrasonic transducers have wide fields of applications, such as ultrasonic motors or ultrasonic processing. Commonly PZT is utilized as active material, transforming the electrical voltage to mechanical vibrations. For most applications, PZT is selected because of its excellent properties [4]. The reason that why the commonly used piezoelectric ceramics is replaced by lithium niobate as the piezoelectric material of ultrasonic transducer are that it is a lead free material and the high Curie temperature of $1210^{\circ} \mathrm{C}$ [4]. Besides the above two reason, lithium niobate has good mechanical performance and chemical stability, and its mechanical quality factor is much higher than common PZT.

The $36{ }^{\circ} \mathrm{Y}-\mathrm{Cut} \mathrm{LiNbO}_{3}$ is the commercial available type of $\mathrm{LiNbO}_{3}$. it is closest to the optimal cut $\left(38.9^{\circ}\right)$ for thickness displacement[18], and has the highest resulting ${ }^{d_{33}}$. Therefore, $36^{\circ} \mathrm{Y}^{-\mathrm{Cut}}{ }^{\mathrm{LiNbO}} \mathrm{O}_{3}$ can be chosen as the piezoelectric vibrator of ultrasonic transducer for oil production[5].

\section{The design of lithium niobate transducer}

Piezoelectric vibrator is the core part of ultrasonic transducers. The new design structure of piezoelectric vibrator of lithium niobate transducer is shown in Figure 1. Round electrode and ring 
electrode are all made of copper.

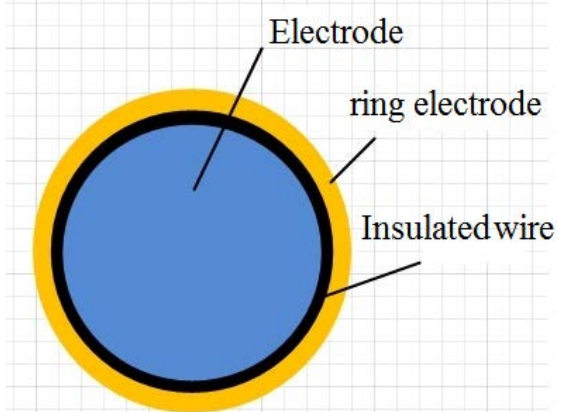

Fig. 1 Schematic diagram of a piezoelectric wafer electrode

The above electrode configuration mode actually use the limited vibration patterns, witch is also called energy trapping vibration mode. Energy-limited vibrator is mainly restricted in the region of the electrode, while the electrode external energy is consumed quickly, therefore, false response and high order harmonic response will not be generated. Thus, the high time of the oscillator noise interference can be eliminated effectively.

The blue round electrode in the middle is connected to the signal line, the area of the blue round electrode is wafer's effective ultrasound radiating area. The ring electrode is connected to aluminum alloy shell. The model of the lithium niobate transducer is shown in Figure 2.

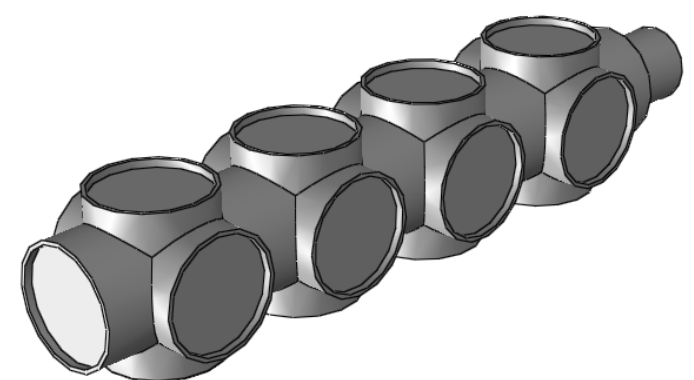

Fig.2 The model of lithium niobate transducer

\section{Harmonic response analysis}

The thickness and radius of the wafer are $0.2 \mathrm{~mm}$ and $15 \mathrm{~mm}$ respectively. The finite model is shown in Figure 3.

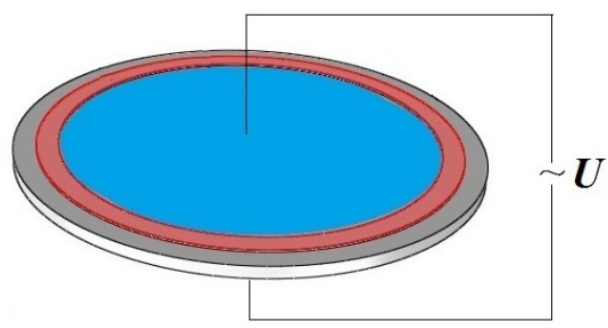

Fig.3 The finite model of the $36 \circ \mathrm{Y}-\mathrm{Cut}^{\mathrm{LiNbO}_{3}}$

The material parameters of lithium niobate crystal are as follows:

Density: $\rho=4640 \mathrm{~kg} / \mathrm{m}^{3}$

Permittivity: $\varepsilon_{11}^{S} / \varepsilon_{0}=44, \quad \varepsilon_{33}^{S} / \varepsilon_{0}=29$

The piezoelectric stress constant: $e_{15}=3.7 \mathrm{C} / \mathrm{m}^{2}, e_{31}=0.2 \mathrm{C} / \mathrm{m}^{2}, e_{33}=1.3 \mathrm{C} / \mathrm{m}^{2}$ 
Elastic constants : $\quad c_{11}^{E}=2.03 \times 10^{11} \mathrm{~N} / \mathrm{m}^{2}, \quad c_{12}^{E}=0.53 \times 10^{11} \mathrm{~N} / \mathrm{m}^{2}, \quad c_{13}^{E}=0.75 \times 10^{11} \mathrm{~N} / \mathrm{m}^{2}$, $c_{33}^{E}=2.45 \times 10^{11} \mathrm{~N} / \mathrm{m}^{2}, \quad c_{44}^{E}=0.60 \times 10^{11} \mathrm{~N} / \mathrm{m}^{2}, \quad c_{66}^{E}=0.75 \times 10^{11} \mathrm{~N} / \mathrm{m}^{2}$ 。

constant damping coefficient can be set about $0.06 \%$. The following formula(1) can be defined for the calculation of important parameters of lithium niobate transducer:

$$
Y=j \omega Q / V, \quad G=\operatorname{Re}(Y), \quad B=\operatorname{Im}(Y)
$$

where $\mathrm{Q}$ is charge value, $\mathrm{V}$ is excitation voltage.

According to the harmonic response analysis of ABAQUS, admittance simulation curve, shown in Figure 4, can be obtained.

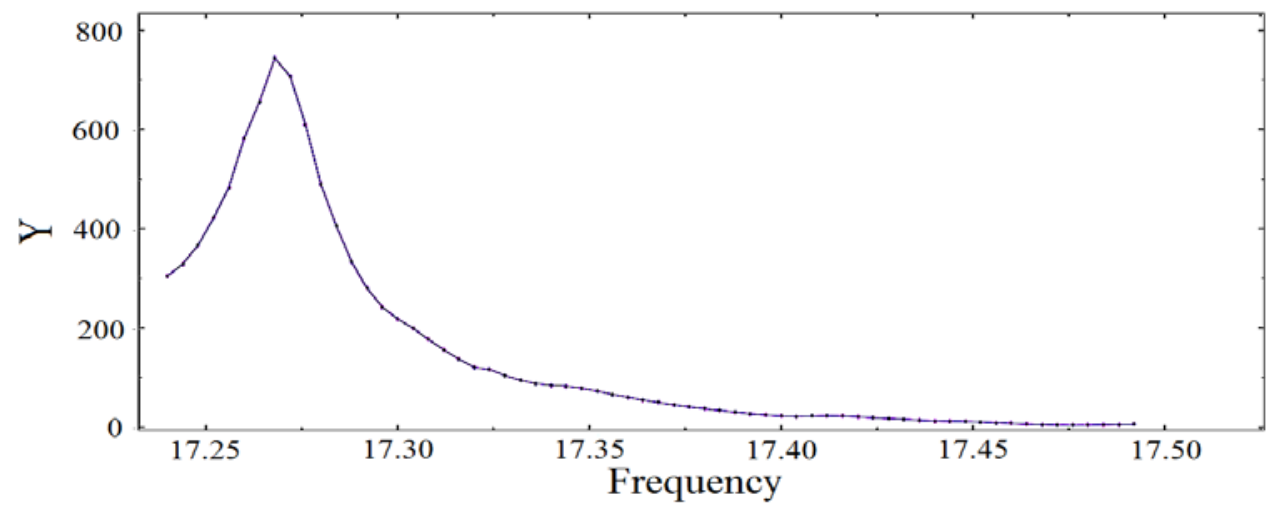

Fig.4 Admittance simulation curve

According to the formula (1) and Figure 3, the fundamental resonant frequency of lithium niobate transduce can be calculated: $f_{s}=17.268 \mathrm{MHz}$; the first half-power point frequency of the resonance point is $f_{1}=17.260 \mathrm{MHz}$, the second half-power point frequency of the resonance point is $f_{2}=17.280 \mathrm{MHz}$. Thus, two important parameters of lithium niobate the transducer can be calculated:

Mechanical quality factor:

$$
Q_{e}=\frac{f_{s}}{f_{2}-f_{1}}=863.4
$$

Effective electromechanical coupling factor:

$$
k_{\text {eff }}=\sqrt{\frac{f_{p}^{2}-f_{s}^{2}}{f_{p}^{2}}}=0.154
$$

The above two important parameters will provide beneficial reference for the design of the transducer and matching circuit. As can be seen in Figure 10, the designed transducer has only one resonance peak in the analysis frequency range $(17.24 \mathrm{MHz}-17.492 \mathrm{MHz})$, which indicates that the designed ultrasonic transducer has no clutter interference near the resonant frequency. According to the above analysis, it is indicated that the structure design of the transducer is reasonable.

\section{Conclusion}

The core part of Enhanced Oil Recovery technique is high-power ultrasonic transducer. Therefore, how to design high-power ultrasonic transducer that satisfy the practical requirement is one of the critical factors that determines its success. In this paper, one high-power ultrasonic transducer made of lithium niobate crystal is designed. According to the harmonic response analysis using ABAQUS, it is indicated that the structure design of the transducer is reasonable.

\section{References}

[1] O.L. Kuznetsov, E.M. Simkin, J. Chillingar, Physical Principles of Vibrational and Acoustic 
Treatment of Oil and Gas Reservoirs, Mir, Moscow, 2001.

[2] Xiaodong Zhang, Xin Chen, Lun Ma, Yanan Zhou, Yue Shi, Gang Yang, Research and application of ultrasonic oil production technique, Ground Water (2013)1-25.

[3] Mohammed Mohsin, Mahmoud Meribout. An extended model for ultrasonic-based enhanced oil recovery with experimental validation. Ultrasonics Sonochemistry.Volume 23, March 2015, Pages 413-423.

[4] Jens Twiefel, Takeshi Morita, Utilizing multilayer lithium niobate elements for ultrasonic actuators, Sens. Actuators, A 166 (2011) 78-82.

[5] Zhenjun Wang, Yuanming Xu, BajracharyaSuman. Research status and development trend of ultrasonic oil production technique in China. UltrasonicsSonochemistry,Volume26, September 2015, Pages 1-8. 